\title{
Cerebral Infarction Associated with 3,4-Methylenedioxymethamphetamine ('Ecstasy') Abuse
}

\begin{tabular}{|l|l|l|}
\hline S. & Shuji & Hanyu $^{\mathrm{a}}$ \\
\hline K. & Kunihiko & Ikeguchi $^{\mathrm{a}}$ \\
\hline H. & Hiroaki & Imai $^{\mathrm{b}}$ \\
\hline N. & Noboru & Imai $^{\mathrm{b}}$ \\
\hline M. & Mitsuo & Yoshida $^{\mathrm{a}}$ \\
\hline
\end{tabular}

${ }^{\mathrm{a}}$ Department of Neurology, Jichi Medical School, and ${ }^{\mathrm{b}}$ Imai Hospital, Ashikaga, Tochigi, Japan

\section{Shuji Hanyu, MD, Department of Neurology, Jichi Medical School, Tochigi-ken 329-04 (Japan)}

3,4-Methylenedioxymethamphetamine (MDMA), more commonly known as 'Ecstasy', is a recreational drug that is gaining popularity worldwide [1]. Although this drug was thought to be safe by recreational users, it has been reported to cause cardiovascular-related deaths [2], hyperthermia, DİC, convulsions, rhabdomyolysis, and acute renal failure [3]. We here report a case of cerebral infarction associated with MDMA abuse.

A healthy 28-year-old man experienced a sudden onset of severe frontal headache with nausea and vomiting, followed by right-sided weakness about $1 \mathrm{~h}$ after he took 2 tablets of Ecstasy in New York. Ten days after the onset, right hand weakness persisted; he therefore had a medical examination in our hospital in Japan. He stated that this was the first time that he had taken MDMA and denied use of other recreational drugs, including alcohol. Medical history was negative for rheumatic fever, previous stroke, hypertension, diabetes, cigarette use, migraine, and hyperlipidemia. There was no family history of stroke or migraine. On physical examination, the patient was afebrile, had a blood pressure of $126 / 70 \mathrm{~mm} \mathrm{Hg}$, and a pulse of 80 beats per min. The results of his general physical and mental status examination were normal. Neurologic examination revealed mild right hemiparesis, right hyperreflexia, and a positive Babinski sign on the right; there were no sensory or visual field abnormalities. Complete blood count, ESR, serum chemistry profile, serum VDRL, prothrombin time, and partial thromboplastin time were normal. Both ECG and echocardiogram showed normal findings. A CT scan showed low attenuation in the left-sided striatocapsular region consistent with an infarction (fig. 1). Drug toxicology studies and angiography were not performed. Over the next few weeks the patient gradually improved without specific therapy. In this case, MDMA use seemed to be the cause of subcortical infarction because (1) the patient lacked other definite stroke risk factors, and (2) a temporal relationship existed between drug abuse and subsequent Itroke symptoms. MDMA is a ring-substituted amphetamine derivative that is chemically related to both hallucinogens and stimulants [4]. Other hallucinogens, amphetamine derivatives or sympathomimetic drugs, such as cocaine [5], methamphetamine [6], phenylpropanolamine [7], phentermine [8] and ephedrine [9] can cause stroke. The clinical course of our case, acute onset of a frontal headache immediately followed by focal neurologic deficits, was similar to that of alkaloidal cocaine-related ischemic stroke, which is considered to be caused by vasospasm [5]. The mechanism of subcortical infarction in our case is unclear, but it might be MDMA-induced vasoconstriction of vessels around the circle of Willis. MDMA potentiates neurotransmission of serotonin [4], and serotonin is the most potent vasoconstrictor amine in the cerebral circulation, especially in large- and medium-sized arteries [10]. MDMA has only rarely produced cerebrovascular accidents [3], and to our knowledge, ischemic stroke has not been described previously in MDMA abusers. The present case, however, serves to illustrate another potential danger of MDMA.

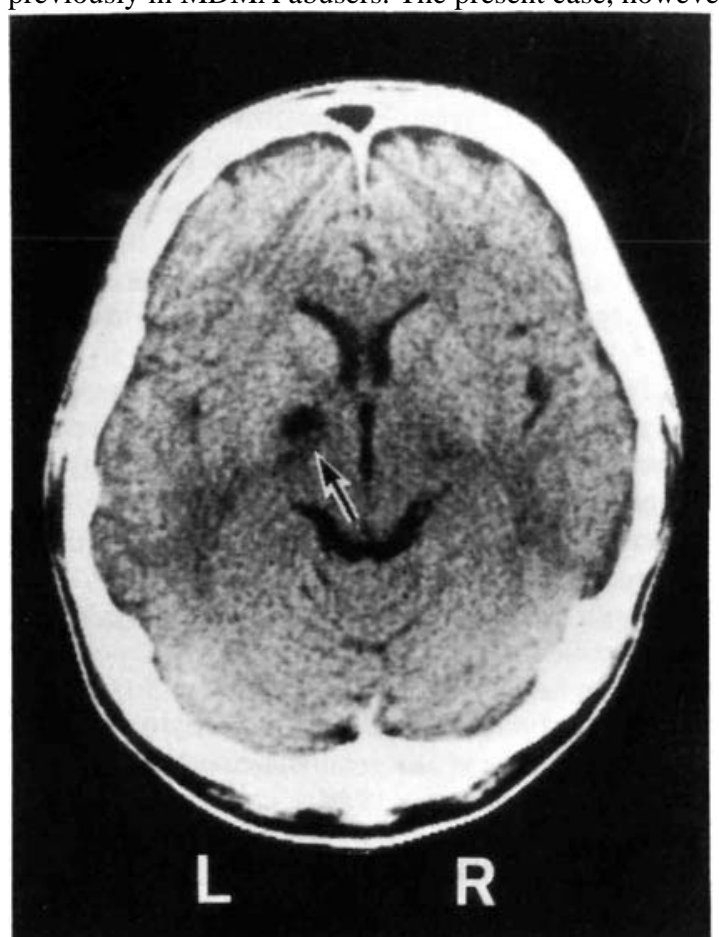

Fig. 1. Computed tomographic scan of brain showing area of low attenuation in left-sided striatocapsular region (arrow). References

Solowiji N, Hall W, Lee N: Recreational MDMA use in Sydney: A profile of'Ecstasy' users and their experiences with the drug. Br J Addict 1992;87: 1161-1172.

Solowiji N, Hall W, Lee N: Recreational MDMA use in Sydney: A profile of Ecstasy' users and heir experiences withe drug. Br J Addict 1992,87:1161-1172.

Henry JA, Jeffrey KJ, Dawling S: Toxicity and deaths from 3,4-methylene-dioxymethamphetamine ('ecstasy'). Lancet 1992;340:384-387.

Johnson MP, Hoffman AT, Nichols DE: Effects of the enantiomers of MDA, MDMA and related analogues on $\left[^{3} \mathrm{H}\right]$ serotonin and $\left[{ }^{3} \mathrm{H}\right] \mathrm{d}$ dopamine release from superfused rat brain slice. Eur J Pharmacol 1986; 132:269-276.

Levine SR, Brust JCM, Futrell N, Ho K-L, Blake D, Millikan CH, Brass LM, Fayad P, Schultz LR, Selwa JF, Welch KMA: Cerebrovascular complications of the use of the 'crack' form of alkaloidal cocaine. N Engl J Med 1990;323:699Levine
704.

Rothrock JF, Rubensteîn R, Lyden PD: Ischemic stroke associated with methamphetamine inhalation. Neurology 1988;38:589-592.

Fallis RJ, Fisher M: Cerebral vaculitis and hemorhage associated with phenylpropanolamine. Neurology 1985:35:405-407.

Kokkinos J, Levine SR: Possible association of ischemic stroke with phentermine. Stroke 1993:24:310-313.

Wooten MR, Khangure MS, Murphy MJ: Intracerebral hemorrhage and vasculitis related to ephedrine abuse. Ann Neurol 1983:13:337-340.

10 Hardebo JE, Edvinsson L, Owman CH, Svendgaad N-A: Potentiation and antagonism of serotonin effects on intracranial and extracranial vessels: Possible implications in migraine. Neurology 1978;28:64-70. 